\title{
OTIMIZAÇÃO DOS PARÂMETROS DE TECIMENTO PARA CONFECÇÃO DE AMANTEIGAMENTO EM CHAPAS DE AÇO AO CARBONO PELO PROCESSO TIG COM ARAME AWS ER309L
}

\author{
(WEAVING PARAMETER OPTIMIZATION FOR BUTTERING ON CARBON STEEL PLATES BY TIG PROCESS WITH \\ AN AWS ER309L WIRE)
}

\author{
Rômulo Queiroz Fratari ${ }^{1}$, Mônica M.A.M. Schvartzman', Américo Scotti ${ }^{1}$ \\ ${ }^{1}$ Universidade Federal de Uberlândia, Centro para Pesquisa e Desenvolvimento de Processos de Soldagem da Faculdade de \\ Engenharia Mecânica, 38400-902,Uberlândia, MG-Brasil,eng romulo05@hotmail.com, ascotti@mecanica.ufu.br \\ ${ }^{2}$ Centro de Desenvolvimento da Tecnologia Nuclear, Serviço de Integridade Estrutural, Av. Presidente Antônio Carlos 6627, 31270- \\ 901, Belo Horizonte, MG-Brasil, monicas@cdtn.br.
}

\begin{abstract}
Resumo
O objetivo do presente trabalho foi avaliar os efeitos dos parâmetros de tecimento e da velocidade de alimentação sobre a formação da primeira camada de amanteigamento com aço inoxidável austenítico, visando a otimização da operação no sentido de maximizar a relação entre espessura da camada e diluição. Os experimentos foram realizados pelo processo TIG com alimentação de arame, variando-se sistematicamente, e garantindo-se a ortogonalidade do planejamento experimental, a amplitude, o tempo de parada lateral e a velocidade de alimentação, permitindo se encontrar equações estocásticas de predições da geometria resultante do depósito. Através de um programa de otimização, procurou-se os parâmetros de regulagem que levassem a maior relação da área depositada pela área fundida. Escolheu-se como restritor a relação reforço por largura, evitando-se revestimentos com alta convexidade e possibilidade de formação de sobreposição lateral de cordão. Soldagens de validação mostraram a robustez e confirmaram a adequabilidade do método de otimização. Com a metodologia empregada foi possível demonstrar a necessidade, para a otimização do cordão de amanteigamento, de se usar a máxima velocidade de alimentação e o mínimo de tempo na parada lateral. A amplitude ideal é um balanço entre os efeitos sobre a área fundida e convexidade, mostrando uma tendência de adquirir um valor maior do que a faixa central.
\end{abstract}

Palavras-chave: Soldagem TIG, Revestimentos, Amanteigamento, Aço Inoxidável.

Abstract: The objective of the present work was to evaluate the effects of the weaving parameters and of the wire feed speed on the formation of a stainless steel buttering layer, aiming the process optimization by the layer height and dilution maximization. The experiments were carried out by the TIG process with wire feeding. Weaving amplitude, lateral dwell time and wire feed speed were systematically varied, through an orthogonal experimental planning, allowing finding stochastic predictive equations of the geometry resulting from the deposits. Through an optimization program, it was sought the tuning parameters to take the largest relationship between deposited and melted areas. It was chosen as restrictor the relationship bead reinforcement by bead width, avoiding deposits with high convexity and possibility of overlay formation. Welding of validation showed the robustness and confirmed the adequacy of the optimization approach. With the employed methodology, it was possible to demonstrate the need of using the fastest wire feed speed and the minimum dwell time for buttering bead optimization. The ideal amplitude is a balance between the effects of it on melted area and bead convexity, presenting a tendency for taking a value greater than the one in the middle of the central range.

Key-words: TIG welding; buttering; stainless steel.

\section{Introdução}

A soldagem de revestimento pode ser definida como a deposição de um material de adição dissimilar sobre uma superfície metálica para se obter propriedades que não são encontradas no material de base [1]. Essa soldagem tem por objetivo, geralmente, um aumento na resistência à abrasão e/ou

(Recebido em 29/07/2009; Texto final em 13/08/2010).

Artigo originalmente publicado no COBEF 2009,

Belo Horizonte, Minas Gerais, Abril de 2009 corrosão do metal base. Segundo Linnert [2], as razões para se efetuar um revestimento de superfície por soldagem podem ser resumidas da seguinte maneira:

(A) Reconstrução do material de base para reparar a forma e ajustar as dimensões de uma peça;

(B) Amanteigamento de uma superfície de um metal de base com camadas de um material dissimilar, que servirá como camada de transição quando a junta subseqüente for executada sobre a camada amanteigada;

(C) Endurecimento superficial com uma camada de composição dissimilar que, por si só, propicia aumento na resistência ao desgaste;

(D) Revestimento do metal de base com a deposição de uma 
camada com composição química dissimilar, que proporcionará ao metal de base diferentes propriedades químicas, físicas e/ ou metalúrgicas, tais como resistência à corrosão, propriedades magnéticas e elétricas;

Assim, um caso particular de revestimentos por soldagem é a técnica de amanteigamento, que pode ser realizada por várias razões. Por exemplo, alguns ferros fundidos, em soldagem pela técnica a frio, muitas vezes exigem uma camada de ferro quase puro (como um aço ABNT 1005) antes de depositar níquel, devido ao grafite impedir a adesão do níquel diretamente [3]. Outra razão seria a possibilidade de acomodar tensões que seriam geradas na interface sobreposta por um material de alta resistência e baixa ductilidade, como, por exemplo, revestimentos duros. Dessa forma, o material de deposição e a operação de amanteigamento devem possuir algumas características em relação ao substrato:

a) Compatibilidade química com o substrato (por exemplo, não se consegue amanteigar aço com alumínio ou mesmo sobre cobre puro);

b) Composição que combinada com a do substrato não resulte em soldas com susceptibilidades a trincas;

c) Alta ductilidade (no caso de se procurar reduzir as tensões geradas pelos passes de união entre os dois componentes);

d) Baixa diluição, através da seleção de parâmetros (considerando que muitas vezes os custos dos materiais de amanteigamento são superiores ao do substrato);

e) ZAC no substrato não susceptível a trincas (conseguida pelo controle dos parâmetros, como, por exemplo, fazendo préaquecimento, ou conseguindo o revenimento da região grosseira - "o tempering bead").

Os fatores que podem influenciar as características de um cordão de amanteigamento (diluição, penetração central, penetração lateral e altura de reforço) são variáveis correlacionadas de forma dependente com o processo (nível de corrente, tipo de gás de proteção, diâmetro do eletrodo, comprimento do arco, distância tocha-peça, posição de soldagem, configuração da junta, velocidade de soldagem, taxa de deposição do material de adição, etc.). Em especial, sabe-se que o tecimento ou oscilação da fonte de calor tem ação preponderante sobre o amanteigamento. $\mathrm{O}$ tecimento é normalmente caracterizado por parâmetros de regulagem, sendo os principais a freqüência e amplitude de oscilação e os tempos de parada central e lateral.

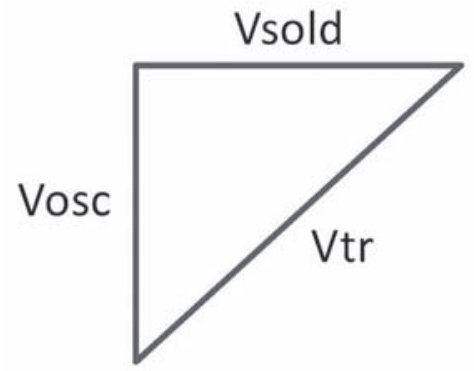

Figura 1: Relação vetorial entre as velocidades de deslocamento longitudinal $\left(\mathrm{V}_{\text {sold }}\right)$ e de oscilação $\left(\mathrm{V}_{\text {osc }}\right)$, resultando na velocidade transversal $\left(\mathrm{V}_{\mathrm{tr}}\right)$ com que a tocha se desloca de um lado para o outro na junta
A velocidade de deslocamento transversal da fonte de calor $\left(\mathrm{V}_{\mathrm{tr}}\right)$, apesar de não regulada no processo de tecimento, é uma variável de grande importância, pois é ela que determina a real distribuição do calor imposto. Como ilustrado pela Figura 1, $\mathrm{V}_{\text {tr }}$ é a resultante vetorial da velocidade de soldagem $\left(\mathrm{V}_{\text {sold }}\right)$ e da velocidade de oscilação $\left(\mathrm{V}_{\text {osc }}\right)$. Esta última, por sua vez, é dependente dos parâmetros de oscilação (amplitude (A), frequiência (f) e tempo de parada lateral $\left(\mathrm{t}_{\mathrm{pl}}\right)$ ), conforme a Eq.1 [4]. A Eq. 2, obtida pelo teorema de Pitágoras, quantifica a relação entre as três velocidades envolvidas.

$$
\begin{aligned}
& V_{\text {osc }}=2 f A / 1-2 f t_{p l} \\
& V_{t r}{ }^{2}=\left(\frac{2 f A}{1-2 f t_{p l}}\right)^{2}+V_{\text {sold }}{ }^{2}
\end{aligned}
$$

Outra variável do tecimento que pode influenciar o amanteigamento é o volume depositado de material por unidade de comprimento da solda. Este volume é representado pela razão entre a velocidade de alimentação e a velocidade de soldagem. Para variar o volume depositado de material por unidade de comprimento da solda, deve-se variar a velocidade de alimentação $\left(\mathrm{V}_{\text {alim }}\right)$ para uma dada velocidade de soldagem caso não se queira que o volume do amanteigamento seja menor.

Assim, todos parâmetros de regulagem da técnica de tecimento influem na $\mathrm{V}_{\mathrm{tr}}$. Além disto, existe correlação entre os próprios parâmetros de tecimento. Por exemplo, um aumento da frequiência altera a relação entre $t_{p l}$ e o tempo de oscilação, da mesma forma que faz o aumento da amplitude para uma mesma freqüência. Fica difícil, então, estudar o efeito de cada parâmetro de tecimento sobre a formação do cordão, já que não há ortogonalidade entre eles (independência). Desta forma, neste trabalho vai se procurar estudar o efeito das variáveis de regulagem da técnica de tecimento sobre a formação do cordão de amanteigamento, mas procurando uma forma de isolar os parâmetros dependentes entre si.

Um outro aspecto importante na operação do amanteigamento é a necessidade de se minimizar a diluição, pois além ser normalmente de maior valor econômico, o material de adição do tecimento tem as propriedades desejadas melhores do que do substrato. Não há sentido, então, misturá-los. Por outro lado, é necessário ter uma alta produção, para tornar a operação viável economicamente. Porém, normalmente produção está relacionada com aumento da energia de soldagem, que leva à maior diluição. Desta forma, um segundo objetivo deste trabalho é também otimizar a regulagem dos parâmetros de tecimento para se conseguir uma relação máxima da área de reforço pela área fundida (quanto maior a área de reforço, maior a produção e, quanto menor a área fundida, menor a diluição), mas sempre garantido que os cordões não apresentem defeitos geométricos, como convexidade excessiva e sobreposição lateral de cordão (conhecido também por "overlap"). 


\section{Metodologia}

Para simular a operação de amanteigamento, se propôs depositar cordões sobre chapa de aço carbono na posição plana, pelo processo TIG com alimentação de arame. A idéia foi definir os parâmetros de interesse para a análise da influência do tecimento sobre as características do cordão de amanteigamento de modo a possuir a maior independência possível entre si, como será explicado adiante. Uma vez definidos, através de um planejamento experimental por fatorial completo $\left(3^{3}\right)$, e após a realização das soldagens e das medições e cálculos necessários, equações preditivas seriam obtidas (pelo método de regressão linear) para os parâmetros de saída desejados (definidos também a seguir). Finalmente, de posse dessas equações, uma otimização do processo (em função dos parâmetros de entrada tomados) seria realizada, objetivando a maximização da equação preditiva objetiva, desde que as restrições fossem obedecidas. Para confirmar a metodologia, validação da otimização seria feita pela realização de novas soldas e medições e cálculos de fatores.

\subsection{Definição dos fatores de entrada}

A velocidade de alimentação do arame é um fator totalmente independente no processo TIG. Pode-se variá-lo, dentro de uma faixa operacional, sem alterar qualquer outro parâmetro. Já se a frequiência, o tempo de parada lateral e a amplitude fossem eleitos fatores ao mesmo tempo, ao se variar a amplitude para uma dada freqüência e tempo de parada lateral, a velocidade transversal mudaria, o que mascararia os resultados (o efeito não seria da amplitude e/ou da freqüência, por exemplo, mas sim deles conjuntamente com a da velocidade transversal). Desta forma, decidiu-se manter as velocidades de soldagem e transversal constantes (permitindo que o calor fornecido ao cordão durante o deslocamento de um lado ao outro seja o mesmo) e usar como fatores a amplitude e o tempo de parada lateral, deixando-se que a frequiência (f) fosse uma variável regulável apenas para permitir se alcançar a velocidade transversal desejada, para cada regulagem de amplitude e/ou tempo lateral. Adotou-se então, a amplitude de oscilação $(\mathrm{A})$, o tempo de parada lateral $\left(\mathrm{t}_{\mathrm{pl}}\right)$ e a velocidade de alimentação do arame $\left(\mathrm{V}_{\text {alim }}\right)$ como fatores de entrada para a análise da influência sobre as características do cordão de amanteigamento.

Visto que as velocidades de deslocamento transversal $\left(\mathrm{V}_{\mathrm{tr}}\right)$ e de soldagem $\left(\mathrm{V}_{\text {sold }}\right)$ foram mantidas constantes, a velocidade de oscilação $\left(\mathrm{V}_{\text {osc }}\right)$, definida pela Eq. 1, também permaneceu constante. A Eq. 3 estabelece a relação para se encontrar " $f$ " entre duas situações diferentes (quando se quer variar a amplitude e/ ou o tempo de parada lateral).

$$
V_{o s c 1}=V_{o s c 2} \quad \text { ou } \quad 2 F_{1} A_{1} / 1-2 F_{1} t_{p l 1}=2 F_{2} A_{2} / 1-2 F_{2} t_{p l 2}
$$

\subsection{Especificação dos fatores de saída (respostas)}

O objetivo é obtenção de revestimentos com alta deposição e baixa diluição. Alta deposição é representada pela área de reforço (ar), enquanto a diluição é diretamente proporcional à área fundida (af), uma vez que a área de reforço é constante. Por isto deve-se otimizar (maximizar) a relação ar/af . Entretanto algumas restrições devem ser levadas em consideração:

\section{a) Convexidade:}

Uma vez que objetiva-se a obtenção de um cordão que possa ser aplicado na preparação de uma camada de amanteigamento, a alta convexidade do mesmo é prejudicial por impedir a adoção de cordões paralelos e sobrepostos, o que poderia causar falta de fusão. Uma das formas de se medir a convexidade de um depósito é através do ângulo de concordância entre metal depósito e o substrato, conhecido como ângulo de molhabilidade. Em função dos cordões apresentarem perfis irregulares e variáveis ao longo de seu comprimento, uma medição da convexidade pelo ângulo se torna difícil. Outra forma seria utilizar as dimensões lineares de "r" e "l" (reforço e largura), pois, de uma forma geral, pode-se esperar que quanto menor a relação $\mathrm{r} / \mathrm{l}$, menor será a convexidade do cordão. Por outro lado, pode haver situações em que essa relação não se aplicaria. Nesse caso, devido à amplitude de tecimento, pode acontecer do reforço aumentar quando a área depositada for maior (maior relação r/l) sem corresponder a uma variação na convexidade. Entretanto, essas situações só ocorreriam para valores muito altos de convexidade (quando o aumento do volume não conseguiria mais alterar o ângulo de molhabilidade), fato não incidente no presente trabalho.

\section{b) Formação de "sobreposição lateral do cordão"}

A "sobreposição lateral do cordão" consiste na formação de cordões com ângulos de concordância entre depósito e substrato menores que $90^{\circ}$. Cordões com a formação de "sobreposição lateral do cordão" são indesejáveis por impedirem a ligação do cordão que seria depositado ao lado. Assim, deve-se evitar sua ocorrência. Observou-se que há uma relação também entre r/l e a "sobreposição lateral do cordão", ou seja, a "sobreposição lateral do cordão" sempre ocorre quando a molhabilidade do cordão no substrato é baixa. Desta forma, para se prevenir deste problema pode-se usar o mesmo parâmetro para evitar alta convexidade.

\subsection{Especificação dos parâmetros de soldagem fixos}

Algumas variáveis do processo (nível de corrente, tipo de gás de proteção, diâmetro do eletrodo, comprimento do arco, distância tocha-peça, posição de soldagem e velocidade de soldagem) e uma variável dependente de outras no tecimento (velocidade transversal) foram fixadas (Tabela 1) objetivando o estudo isolado do efeito das variáveis independentes de tecimento (amplitude e tempo de paradas lateral) e da velocidade de alimentação, a três níveis cada (totalizando 27 cordões soldados) sobre as características do cordão de amanteigamento (área de reforço, área fundida, reforço e largura). O ângulo de ataque foi escolhido para propiciar menores penetrações e condições satisfatórias de proteção gasosa. A entrada do arame (pela frente do arco através da poça), assim como o ângulo de entrada do arame, foram selecionados para que o arame se fundisse no momento em que tocasse a poça de fusão, diminuindo a 
Tabela 1: Parâmetros de regulagem do processo TIG com alimentação de arame, para cordão sobre a chapa na posição plana, cujos níveis foram fixos durante a execução do planejamento

\begin{tabular}{|l|c|}
\hline \multicolumn{1}{|c|}{ Fatores fixos } & Valores \\
\hline Velocidade transversal do tecimento & $24 \mathrm{~mm} / \mathrm{s}$ \\
\hline Velocidade de soldagem & $2,0 \mathrm{~mm} / \mathrm{s}$ \\
\hline Intensidade de corrente & $180 \mathrm{~A}$ \\
\hline Ângulo de ataque da tocha & Arngulo $=10^{\circ}$ empurrando \\
\hline Gás de Proteção & Argônio comercial $(99,995 \%)$ \\
\hline Vazão & Aço ABNT 1020 \\
\hline Tipo de material de base (substrato) & AWS ER309L \\
\hline Tipo do material de adição (depósito) & $1,0 \mathrm{~mm}$ \\
\hline Diâmetro dos arames de adição & AWS EWTh-2 /3,2 mm \\
\hline Eletrodo TIG & \\
\hline Ângulo de entrada da alimentação & $70^{\circ}$ \\
\hline & \\
\hline
\end{tabular}

Figura 2. Esquema para estratificação das áreas dos cordões para medição por tratamento de imagens através de delimitação das áreas no Photoshop: à esquerda, seção transversal sem tratamento; no centro, área fundida em destaque; à direita, área de reforço em destaque

quantidade de calor que seria fornecida para a fusão do metal base.

\section{Desenvolvimento Experimental}

Foram usados para se fazer cordões barras de aço carbono ABNT 1020, com dimensões de $200 \mathrm{~mm}$ de comprimento, $50 \mathrm{~mm}$ de largura e espessura de $6,4 \mathrm{~mm}$. O processo TIG foi utilizado na forma convencional, com alimentação automatizada de arame, corrente contínua e polaridade direta (CC-). A fonte de soldagem foi uma transistorizada com chaveamento no secundário, trabalhando no modo característica estática de corrente constante. Utilizou-se um alimentador de arame adaptado para as baixas velocidades de alimentação demandadas. Para a movimentação do sistema tocha, utilizou-se uma mesa de coordenadas para soldagem, que possibilitou a realização de depósitos lineares com oscilações, por meio de uma interface na qual se estabelece os valores de amplitude, frequiência, tempo de parada lateral, velocidade de soldagem e trajetória a ser percorrida.

Após a obtenção dos cordões, seções transversais para análise macrográfica foram retiradas a um terço do cordão, á partir do início. Foram, então, embutidas e lixadas até a lixa \#600 e atacadas com NITAL 2\%. As imagens das seções transversais foram obtidas com o auxílio de uma câmera fotográfica digital convencional, acoplada a um sistema de aquisição de imagens. As imagens foram tratadas no programa Adobe Photoshop, utilizando-se as ferramentas lasso, para selecionar a área desejada (ar ou af), e paint bucket, para destacá-la em relação ao restante da imagem. A Figura 2 ilustra uma seção transversal antes do tratamento, a área fundida em destaque e a área de reforço em destaque. Através do software de análise de imagens e após a calibração da unidade de comprimento (ferramenta calibration), de posse da imagem de uma rede com dimensões conhecidas, obtiveram-se os valores das características geométricas do depósito (áreas de reforço e fundida), medidas através de uma seção transversal dos cordões, pela ferramenta particle. Os valores de reforço e largura foram medidos por um paquímetro antes do seccionamento, sendo que foram realizadas 7 medidas sobre o cordão. Medições desses 2 parâmetros com o paquímetro possuem uma maior confiabilidade, uma vez que é realizado um maior número de medições e ao longo de todo cordão, ao contrário de quando se usa a seção transversal. 


\subsection{Determinação exploratória dos limites dos fatores}

\subsubsection{Velocidade de alimentação $\left(\mathrm{V}_{\text {alim }}\right)$}

Foram realizados ensaios preliminares objetivando a determinação do valor máximo de velocidade de alimentação que possibilitasse a soldagem de forma operacional (evitando o toque do arame com a poça, o que poderia resultar na extinção do arco). Observou-se que para valores de velocidade de alimentação acima de $55 \mathrm{~mm} / \mathrm{s}$ o calor fornecido pelo arco não foi suficiente para fundir o arame antes que ele alcançasse a chapa, resultando no contato do arame com a poça e conseqüente extinção do arco. Dessa forma, adotou-se o valor de $52,5 \mathrm{~mm} / \mathrm{s}$ como valor máximo de velocidade de alimentação do arame. Devido à necessidade de se utilizar três valores de velocidade de alimentação normalizados, utilizou-se o limite superior como ponto de partida $(52,5 \mathrm{~mm} / \mathrm{s}$ real $)$ e os valores de $23,5 \mathrm{~mm} / \mathrm{s}$ e 38 $\mathrm{mm} / \mathrm{s}$ como os demais valores. O valor mínimo $(23,5 \mathrm{~mm} / \mathrm{s})$ foi adotado por ser aquele que propiciou a obtenção de cordões com volume aceitável, a partir de ensaios preliminares.

\subsubsection{Frequiência (f), amplitude (A) e tempo de parada lateral $\left(\mathbf{t}_{\mathrm{p})}\right)$}

Inicialmente, foram realizados ensaios com A de $6 \mathrm{~mm}$ e $\mathrm{t}_{\mathrm{pl}}$ de $0,15 \mathrm{~s}$. Valores de $\mathrm{f}$ muito baixos (menores que $1 \mathrm{~Hz}$ ) resultaram em cordões de alta convexidade. Já para A de $12 \mathrm{~mm}$, valores de $\mathrm{F}$ abaixo de $0,75 \mathrm{~Hz}$ resultaram em cordões muito irregulares com regiões não fundidas. Dessa forma, adotou-se $\mathrm{F}$ de $1 \mathrm{~Hz}$ para A de $12 \mathrm{~mm}$ e tpl nulo. Os valores de f para as demais situações foram calculados a partir da Eq. 3, visto que o objetivo foi o de manter constante a velocidade de deslocamento transversal e, conseqüentemente, a velocidade de oscilação (pois a velocidade de soldagem também é mantida constante). Como o equipamento aceita valores de frequiência de 0 a $5 \mathrm{~Hz}$, a menor amplitude adotada, em função dos valores de freqüências, foi de $3 \mathrm{~mm}$. Valores abaixo de $3 \mathrm{~mm}$ resultariam em freqüências que ultrapassariam o limite de $5 \mathrm{~Hz}$.

Outro ponto importante diz respeito ao $t_{\mathrm{pl}}$ não exceder o limite de $40 \%$ do período total de oscilação $(\mathrm{T}=1 / \mathrm{f})$. Dessa forma, para um $t_{p l}$ de $0,15 \mathrm{~s}$, f não pode exceder o valor de $2,66 \mathrm{~Hz}$, enquanto para um $t_{p l}$ de $0,30 \mathrm{~s}$ f não pode ultrapassar $1,33 \mathrm{~Hz}$. A Tabela 2 mostra os valores de $A$ e de $t_{p 1}$ que foram adotados, com as respectivas freqüências de regulagem para obtê-los.

\subsection{Planejamento experimental}

O planejamento experimental adotado foi um fatorial completo de três variáveis a três níveis $\left(3^{3}\right)$. A Tabela 3 mostra as variáveis independentes envolvidas neste estudo e respectivos níveis. As análises estatísticas dos resultados foram feitas com o auxílio de um software comercial, possibilitando a escolha do planejamento, das variáveis independentes (e possíveis interações) e da variável dependente em questão.
Tabela 2: Valores de amplitude (A) e tempo de parada lateral $\left(t_{p p}\right)$ adotados e suas respectivas frequiências (f) de regulagem

\begin{tabular}{|c|c|c|}
\hline $\mathrm{A}(\mathrm{mm})$ & $\mathrm{t}_{\mathrm{pl}}(\mathrm{s})$ & $\mathrm{f}(\mathrm{Hz})$ \\
\hline 12,0 & - & 1,0 \\
\hline 7,5 & - & 1,6 \\
\hline 3,0 & - & 4,0 \\
\hline 12,0 & 0,15 & 0,77 \\
\hline 7,5 & 0,15 & 1,08 \\
\hline 3,0 & 0,15 & 1,82 \\
\hline 12,0 & 0,30 & 0,625 \\
\hline 7,5 & 0,30 & 0,82 \\
\hline 3,0 & 0,30 & 1,18 \\
\hline
\end{tabular}

Tabela 3: Fatores e níveis utilizados no planejamento experimental

\begin{tabular}{|l|c|c|c|}
\hline & -1 & 0 & 1 \\
\hline Amplitude (mm) & 3 & 7,5 & 12 \\
\hline Tempo de parada lateral (s) & 0 & 0,15 & 0,30 \\
\hline Velocidade de alimentação (mm/s) & 23,5 & 38,0 & 52,5 \\
\hline
\end{tabular}

Após a utilização do método de regressão linear, evidenciou os coeficientes dos termos das equações preditivas desejadas (usando os fatores na forma normalizada, de $-1 \mathrm{a}+1$ ). Foram obtidas equações preditivas (objetiva e restritiva) para ar, af, $\mathrm{r}$ e $1 \mathrm{em}$ separado e depois as equações foram arranjadas de modo a se obter ar/af (objetiva) e r/l (restritiva). Adquiriram-se também equações preditivas a partir dos valores de ar/af e r/l. As análises estatísticas puderam comprovar quais as melhores equações preditivas a serem adotadas, se as equações de $r, 1$, ar e af obtidas em separado ou as equações obtidas para as relações $\mathrm{r} / \mathrm{l}$ e ar/ af, em função de valores tais como, desvio padrão, tendências estatísticas, coerência das equações com o esperado pela teoria.

\subsection{Otimização dos parâmetros}

A otimização da equação objetiva (ar/af), respeitando o restritor (r/l), foi feita por meio de um comando de otimização do software Matlab®, chamado "fminimax", que calcula o mínimo ou o máximo de uma função de multivariáveis por meio de uma estimativa inicial. Para tal, criaram-se dois comandos com extensão "m", um onde se insere a função que se queira fazer a otimização (fminimax) e outro onde se estabelece a função da restrição necessária (restineqx). O programa faz a minimização de um fator, sendo, assim, necessário que a equação objetiva seja elevada a -1. Já o programa propriamente dito de otimização é composto pelo valor da estimativa inicial, dos limites de cada variável e de opções da otimização, tais como número máximo de iterações, tolerância e o tipo de otimização. 
Tabela 4: Características geométricas relativas aos ensaios para determinação da influência dos parâmetros de oscilação

\begin{tabular}{|c|c|c|c|c|c|c|c|c|c|}
\hline $\mathrm{N}$ & $\mathrm{A}(\mathrm{mm})$ & $\mathrm{t}_{\mathrm{pl}}(\mathrm{s})$ & $\mathrm{V}_{\text {alim }}(\mathrm{mm} / \mathrm{s})$ & $\mathrm{ar}\left(\mathrm{mm}^{2}\right)$ & $\mathrm{af}\left(\mathrm{mm}^{2}\right)$ & $\mathrm{ar} / \mathrm{af}$ & $\mathrm{r}(\mathrm{mm})$ & $1(\mathrm{~mm})$ & $\mathrm{r} / 1$ \\
\hline 1 & 3 & 0 & 23,5 & 8,16 & 14,39 & 0,567 & 1,56 & 7,14 & 0,218 \\
\hline 2 & 3 & 0 & 38,0 & 3,41 & 7,57 & 1,772 & 2,70 & 6,39 & 0,423 \\
\hline 3 & 3 & 0 & 52,5 & 19,36 & 5,14 & 3,767 & 3,64 & 6,11 & 0,596 \\
\hline 4 & 3 & 0,15 & 23,5 & 7,96 & 13,24 & 0,601 & 1,31 & 7,66 & 0,171 \\
\hline 5 & 3 & 0,15 & 38 & 11,20 & 3,24 & 3,457 & 2,59 & 6,14 & 0,422 \\
\hline 6 & 3 & 0,15 & 52,5 & 15,58 & 3,80 & 4,100 & 3,29 & 6,18 & 0,532 \\
\hline 7 & 3 & 0,3 & 23,5 & 8,26 & 11,46 & 0,721 & 1,63 & 7,83 & 0,208 \\
\hline 8 & 3 & 0,3 & 38 & 12,58 & 6,33 & 1,987 & 2,35 & 7,09 & 0,332 \\
\hline 9 & 3 & 0,3 & 52,5 & 18,43 & 4,00 & 4,608 & 3,49 & 6,49 & 0,539 \\
\hline 10 & 7,5 & 0 & 23,5 & 8,99 & 11,81 & 0,761 & 1,36 & 9,56 & 0,142 \\
\hline 11 & 7,5 & 0 & 38 & 12,46 & 7,75 & 1,608 & 2,04 & 9,27 & 0,220 \\
\hline 12 & 7,5 & 0 & 52,5 & 17,24 & 5,76 & 2,993 & 2,47 & 8,89 & 0,278 \\
\hline 13 & 7,5 & 0,15 & 23,5 & 8,80 & 12,66 & 0,695 & 1,07 & 11,10 & 0,097 \\
\hline 14 & 7,5 & 0,15 & 38 & 12,22 & 8,46 & 1,444 & 1,76 & 10,52 & 0,167 \\
\hline 15 & 7,5 & 0,15 & 52,5 & 17,52 & 4,25 & 3,995 & 2,51 & 10,02 & 0,250 \\
\hline 16 & 7,5 & 0,3 & 23,5 & 7,91 & 14,29 & 0,554 & 1,10 & 11,62 & 0,095 \\
\hline 17 & 7,5 & 0,3 & 38 & 13,36 & 8,73 & 1,530 & 1,65 & 10,82 & 0,152 \\
\hline 18 & 7,5 & 0,3 & 52,5 & 17,73 & 6,17 & 2,842 & 2,39 & 10,27 & 0,233 \\
\hline 19 & 12 & 0 & 23,5 & 13,45 & 7,41 & 1,815 & 1,41 & 12,17 & 0,116 \\
\hline 20 & 12 & 0 & 38 & 15,83 & 6,44 & 2,459 & 1,79 & 11,97 & 0,150 \\
\hline 21 & 12 & 0 & 52,5 & 17,92 & 6,92 & 2,589 & 2,09 & 12,33 & 0,169 \\
\hline 22 & 12 & 0,15 & 23,5 & 8,28 & 11,75 & 0,705 & 0,79 & 14,85 & 0,053 \\
\hline 23 & 12 & 0,15 & 38 & 13,25 & 8,40 & 1,578 & 1,14 & 15,72 & 0,072 \\
\hline 24 & 12 & 0,15 & 52,5 & 19,42 & 5,03 & 3,861 & 1,76 & 14,46 & 0,122 \\
\hline 25 & 12 & 0,3 & 23,5 & 9,12 & 14,36 & 0,635 & 0,88 & 17,10 & 0,051 \\
\hline 26 & 12 & 0,3 & 38 & 14,14 & 8,19 & 1,727 & 1,18 & 15,63 & 0,075 \\
\hline 27 & 12 & 0,3 & 52,5 & 16,59 & 5,87 & 2,826 & 1,87 & 15,05 & 0,124 \\
\hline
\end{tabular}

\section{Resultados e Discussão}

A Tabela 4 mostra a identificação das amostras $(\mathrm{N})$ com as correspondentes variáveis independentes (amplitude, A, tempo de parada lateral, $\mathrm{t}_{\mathrm{pl}}$, e velocidade de alimentação, $\mathrm{V}_{\text {alim }}$ ) e os valores obtidos para área de reforço (ar), área fundida (af), reforço (r), largura (l) e as relações r/l e ar/af.

\subsection{Determinação da equação preditiva para a relação restritiva $\mathrm{r} / \mathrm{l}$}

Foram obtidas equações para o reforço, a largura em relação r/l. A análise dos resíduos estatísticos para as equações obtidas a partir das medições realizadas pelo programa mostrou distribuição aleatória, sem tendências e os valores observados possuíram boa concordância com os valores preditos.

O esperado é de que com um aumento na amplitude o reforço diminua e a largura aumente, pelo fato de se aquecer melhor nas laterais dos cordões e aumentar a molhabilidade dos mesmos com o material de base (maior espalhamento da poça fundida). Com relação ao tempo de parada lateral, espera-se que quanto maior o $\mathrm{t}_{\mathrm{pl}}$ também maior será a largura e menor será o reforço (maior será o tempo que a poça fundida permanece na lateral, aumentando também o espalhamento da poça fundida).
Quanto à velocidade de alimentação, se aumentada, novamente se prevê que o reforço e largura também aumentem, mas pode acontecer de não serem estes aumentos proporcionais, pois há uma maior quantidade de material sendo adicionada e, conseqüentemente, menor será o espalhamento da poça de fusão. Dessa forma, espera-se que um aumento na velocidade de alimentação acarrete em maiores convexidades (seguindo o conceito de quanto maior a relação r/l maior será a convexidade).

Pela comparação entre as equações obtidas em separado e a equação obtida para a relação $r / 1$, observou-se que o comportamento em ambas as situações foi similar ao esperado pela teoria, onde um aumento na amplitude resultou em aumento na largura e queda no reforço e, por conseguinte, queda na relação $r / 1$. Da mesma forma, um aumento no $t_{p l}$ causou queda no reforço e aumento na largura, diminuindo a relação r/l enquanto um aumento na $\mathrm{V}_{\text {alim }}$ resultou em aumento na relação r/l. Entretanto, uma análise dos gráficos de valores preditos $\mathrm{x}$ observados evidenciou que a equação obtida para a relação r/l foi menos tendenciosa que as equações obtidas para $r$ e 1 em separado.

Adotou-se, portanto, como equação preditiva para a relação restritiva $\mathrm{r} / \mathrm{l}$, aquela obtida a partir das medições realizadas para os valores de r/l, conforme a Eq. 4. 


$$
\begin{aligned}
& r / L=0,0475-0,034 A+0,003 A^{2} \\
& -0,1857 t_{p l}+0,0149 V_{a \lim }-0,0011 A V_{a \lim }
\end{aligned}
$$

\subsection{Definição da restrição (r/l)}

A relação r/l obtida variou de valores em torno de 0,05 (Figura 3a) a 0,60 (Figura $3 b$ ). Para definir um valor limite de $r / 1$ para ser adotado como restritor, tanto para alta convexidade como para ocorrência de "sobreposição lateral do cordão", visualizouse as imagens das seções transversais dos cordões e definiu-se subjetivamente geometrias como aceitáveis e não aceitáveis. Assim, como o objetivo da restrição é minimizar a convexidade do cordão, bem como anular a possibilidade de ocorrência de "sobreposição lateral do cordão", escolheu-se arbitrariamente (pela visualização) como condição restritiva, situações onde a relação r/l não atingisse valores maiores ou iguais a 0,15 .
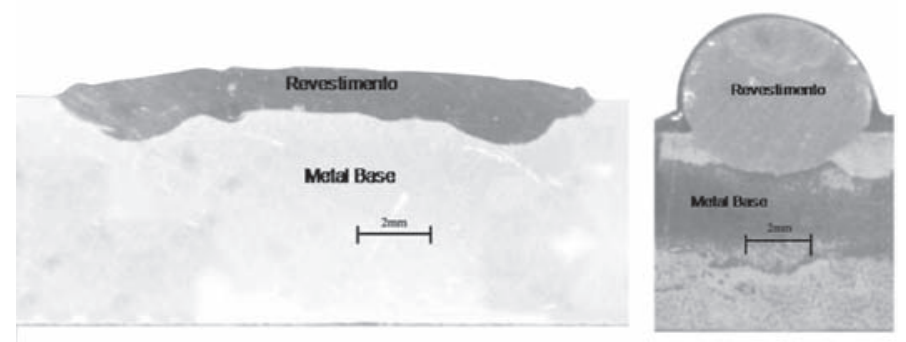

Figura 3: Seções transversais de dois cordões: à esquerda $\left(\mathrm{A}=12 \mathrm{~mm} ; \mathrm{t}_{\mathrm{pl}}=0,15 \mathrm{~s} ; \mathrm{V}_{\mathrm{alim}}=23,5 \mathrm{~mm} / \mathrm{s}\right)$, com $\mathrm{r} / \mathrm{l}=0,05$ (baixíssima convexidade e inexistência de "sobreposição lateral do cordão"); à direita ( $\mathrm{A}=3 \mathrm{~mm} ; \mathrm{t}_{\mathrm{pl}}=0 \mathrm{~s} ; \mathrm{V}_{\text {alim }}=52,5$ $\mathrm{mm} / \mathrm{s}$ ), com $\mathrm{r} / \mathrm{l}=0,6$ (alta convexidade e início da ocorrência de "sobreposição lateral do cordão")

\subsection{Obtenção da equação preditiva objetiva de ar/af}

Obtiveram-se equações para os valores de ar, af e para a relação ar/af. Essas equações apresentaram razoável ajuste $\left(\mathrm{R}^{2}>\right.$

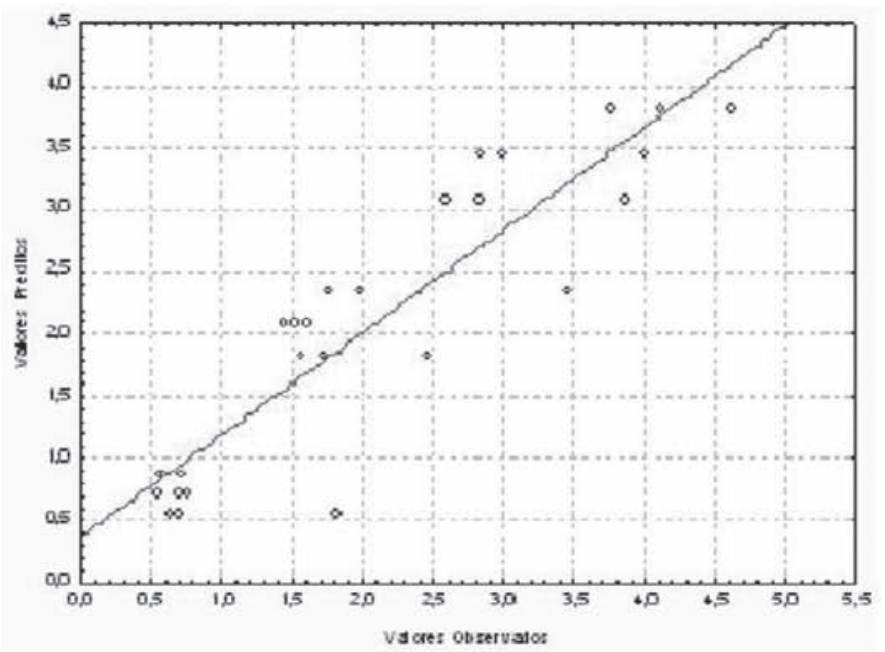

0,81) e boa representabilidade pela análise de resíduo. Optou-se pelo uso do preditor de ar/af, pois esta relação representa melhor o objetivo da maximização do que os valores preditos de ar e af em separado. A Figura 4 evidencia os valores observados $\mathrm{x}$ preditos e análise de resíduo para a relação ar/af. Dessa forma, a equação preditiva objetiva obtida para os valores de ar/af foi adotada (Eq. 5) e utilizada para a otimização do processo.

$$
\frac{a r}{a f}=-1,4897+0,1053 V_{a \lim }-0,0015 A \cdot V_{a \lim }
$$

Pela equação obtida, pode-se verificar que com o aumento na velocidade de alimentação a relação ar/af também aumentou. De fato, com o aumento na velocidade de alimentação a relação ar/af deveria crescer, visto que há um acréscimo na quantidade de material depositado e, consequentemente, parte do calor que seria fornecido para fundir a chapa é retirada para fundir essa maior quantidade de material adicionado (aumenta ar e diminui af). Com relação à amplitude, o incremento em seus valores resultou no aumento da área fundida (aumentou a diluição) e, por conseguinte, houve decréscimo na relação ar/af. Este resultado encontra divergência com dados da literatura. Por exemplo, alguns autores $[5,6]$ citam que altas amplitudes tendem a diminuir a diluição. Esta contradição sugere que o efeito da amplitude é dependente de outros fatores. No presente caso, que trata de TIG com arame frio, acredita-se que na faixa de amplitude estudada, o espalhamento do cordão tenha melhorado a transferência de calor do arco para chapa, propiciando uma maior diluição. Finalmente, quanto ao tempo de parada lateral, um aumento em seus valores não induziu a mudanças na relação ar/af. Da mesma forma, o esperado era que o aumento no tempo de parada lateral não alterasse a relação ar/af, uma vez que, há um maior espalhamento da poça fundida, aumentando a largura e diminuindo tanto a penetração quanto o reforço.

\subsection{Otimização}

De posse das equações preditivas (restritiva e objetiva), pôde-se enunciar uma relação otimizada, na qual se tem a

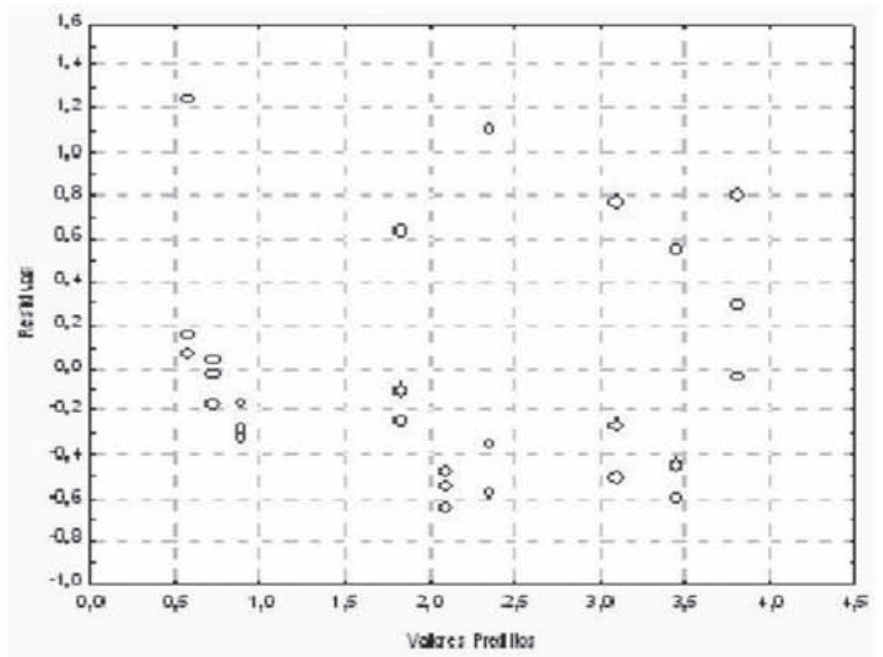

Figura 4: Valores observados x valores preditos e resíduos para a relação ar/af 
Tabela 5: Valores de regulagem dos parâmetros de tecimento e valores obtidos das características geométricas dos revestimentos para ensaios de validaçào

\begin{tabular}{|c|c|c|c|c|c|c|c|c|c|}
\hline \multirow{2}{*}{ Amostra } & \multicolumn{4}{|c|}{ Regulagem } & \multicolumn{4}{c|}{ Medidos } & \multicolumn{3}{c|}{ Calculados } \\
\cline { 2 - 11 } & $\mathrm{A}(\mathrm{mm})$ & $\mathrm{t}_{\mathrm{pl}}(\mathrm{s})$ & $\mathrm{V}_{\text {alim }}(\mathrm{mm} / \mathrm{s})$ & $\mathrm{ar}\left(\mathrm{mm}^{2}\right)$ & $\mathrm{af}\left(\mathrm{mm}^{2}\right)$ & $\mathrm{r}(\mathrm{mm})$ & $\mathrm{1}(\mathrm{mm})$ & $(\mathrm{r} / \mathrm{l})$ & ar/af \\
\hline AC & 3 & 0 & 52,5 & 21,31 & 7,60 & 3,13 & 7,34 & 0,43 & 3,10 \\
\hline Amostra 3 & 3 & 0 & 52,5 & 19,36 & 5,14 & 3,64 & 6,11 & 0,60 & 3,80 \\
\hline BAR & 12 & 0,3 & 23,5 & 11,34 & 15,49 & 1,04 & 16,26 & 0,06 & 0,73 \\
\hline Amostra 25 & 12 & 0,3 & 23,5 & 9,12 & 14,36 & 0,88 & 17,10 & 0,05 & 0,64 \\
\hline OT & 9,1 & 0 & 52,5 & 20,69 & 6,09 & 2,12 & 11,02 & 0,19 & 3,23 \\
\hline
\end{tabular}

maximização da relação ar/af (alta produção e baixa diluição), mas garantindo que a restrição $\mathrm{r} / \mathrm{l}<0,15$ fosse respeitada (atendendo o efeito de convexidade e "sobreposição lateral de cordão"). O valor da estimativa inicial foi escolhido como os valores de limites inferiores dos fatores, ou seja, 3,0 mm (A), $0 \mathrm{~s}\left(\mathrm{t}_{\mathrm{pl}}\right)$ e $23,5 \mathrm{~mm} / \mathrm{s}\left(\mathrm{V}_{\mathrm{alim}}\right)$. Já o limite superior foi de $12,0 \mathrm{~mm}$ (A), 0,3 s ( $\left.\mathrm{t}_{\mathrm{pl}}\right)$ e $52,5 \mathrm{~mm} / \mathrm{s}\left(\mathrm{V}_{\text {alim }}\right)$, pois não se desejava extrapolar ao envelope estudado. $\mathrm{O}$ número de iterações foi limitado pela equação restritiva (rineqx3), não sendo necessária sua definição. Optou-se por uma tolerância de $95 \%$ de confiabilidade, enquanto o tipo de otimização foi o de minimax. Após rodar o programa, obtiveram-se os valores de $\mathrm{A}, \mathrm{t}_{\mathrm{pl}}$ e $\mathrm{V}_{\text {alim }}$ que maximizaram a relação ar/af. $\mathrm{A} \mathrm{V}_{\text {alim }}$ ótima foi o valor máximo adotado no planejamento $(52,5 \mathrm{~mm} / \mathrm{s})$, enquanto $\mathrm{o}_{\mathrm{pl}}$ ótimo foi $\mathrm{o}$ valor mínimo adotado (nulo). Já a amplitude ótima foi um valor intermediário. A Figura 5 mostra uma imagem da amostra $12 \mathrm{e}$ outra da amostra 21, cujos valores de "A" foram bem distintos. A partir desta figura, pode-se observar que o cordão obtido para $\mathrm{A}=7,5 \mathrm{~mm}$ (amostra 12), apesar de possuir uma boa relação ar/ af, apresentava convexidade além da desejada. Por outro lado, o cordão obtido para $A=12 \mathrm{~mm}$ satisfez a restrição imposta (r/l $<0,15)$, mas com baixo valor para a relação ar/af. Ilustra-se, assim, que a amplitude ótima calculada $(9,1 \mathrm{~mm})$ se encontre entre esse intervalo de amplitude $(7,5-12 \mathrm{~mm})$.
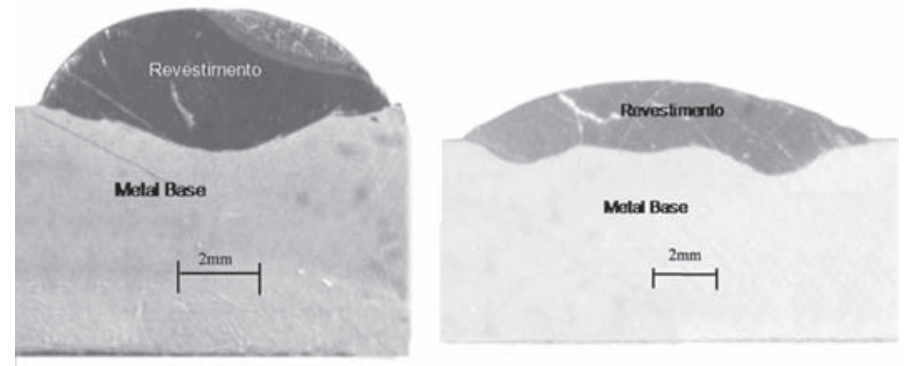

Figura 5: Seção transversal de soldagens feitas para otimização de parâmetros de tecimento: à esquerda, amostra 12 com $\mathrm{A}=7,5 \mathrm{~mm}(\mathrm{ar} / \mathrm{af}=2,99 \mathrm{e} \mathrm{r} / \mathrm{l}=0,28)$; à direita, amostra

$21 \operatorname{com~} \mathrm{A}=12 \mathrm{~mm}(\mathrm{ar} / \mathrm{af}=2,59$ e $\mathrm{r} / \mathrm{l}=0,17)$

\subsection{Validação dos parâmetros otimizados}

Para avaliação da repetibilidade experimental, procurou-se realizar soldagens de replicagem de duas soldagens iniciais, procurando condições paramétricas que levassem à alta convexidade $\left(\mathrm{A}=3 \mathrm{~mm} ; \mathrm{t}_{\mathrm{pl}}=0 \mathrm{~s} ; \mathrm{V}_{\mathrm{alim}}=52,5 \mathrm{~mm} / \mathrm{s}\right)$ e à baixa relação ar/af $\left(A=12 \mathrm{~mm} ; \mathrm{t}_{\mathrm{pl}}=0,3 \mathrm{~s} ; \mathrm{V}_{\mathrm{alim}}=23,5 \mathrm{~mm} / \mathrm{s}\right)$, as quais foram tratadas como AC e BAR, respectivamente. A Tabela 5 mostra a quantificação da avaliação geométrica dos cordões das soldagens, enquanto a Figura 6 ilustra as seções transversais destes cordões. Pôde-se observar em ambos os casos perfis de cordão bem similares, demonstrando a repetibilidade. Mas, por outro lado, a não concordância exata dos valores de ar/af e r/l indicam ser esperado erros aleatórios. Também foi feita uma soldagem na condição encontrada otimizada $\left(A=9,1 \mathrm{~mm} ; \mathrm{t}_{\mathrm{pl}}=0\right.$ $\mathrm{s} ; \mathrm{V}_{\text {alim }}=52,5 \mathrm{~mm} / \mathrm{s}$ ), tratada como OT, para validação do método de otimização dos parâmetros de tecimento. A seção transversal desta soldagem é mostrada na Figura 7. Pode-se observar que a diluição foi baixa (pequena área fundida) enquanto a área de reforço foi alta (alta produção) sem, no entanto, ultrapassar os limites de convexidade.
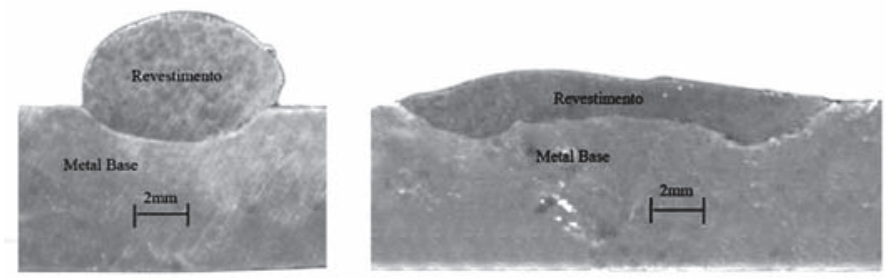

Figura 6. Seções transversais de cordões de validação: à esquerda, amostra $\mathrm{AC}(\mathrm{ar} / \mathrm{af}=3,07 \mathrm{e} \mathrm{r} / \mathrm{l}=0,43)$; à direita, amostra BAR (ar/af $=0,73$ e $r / 1=0,06)$

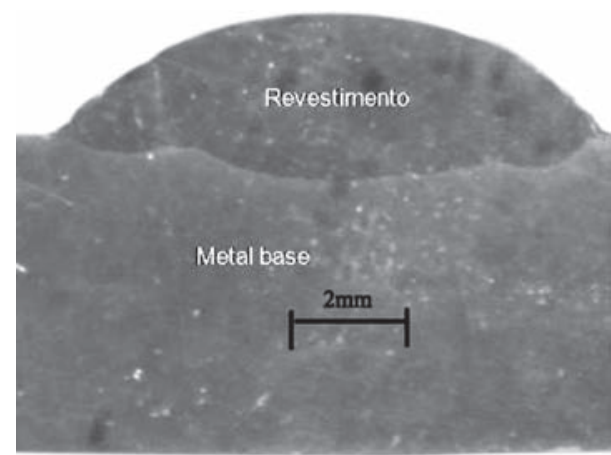

Figura 7: Amostra com regulagem de parâmetros que levassem à condição otimizada (OT), com ar/af = 3,23 e $\mathrm{r} / \mathrm{l}=0,19$ 
Conforme esperado, apesar da amostra AC apresentar um valor elevado para a relação ar/af (> 3), o valor de r/l foi muito alto $(0,43)$ o que comprova a alta convexidade. Com relação à amostra BAR, apesar da baixa convexidade $(\mathrm{r} / \mathrm{l}=0,06)$, o valor de ar/af foi muito baixo $(\approx 0,7)$ em função da alta diluição. Já a amostra OT apresentou um alto valor para a relação ar/ af $(\approx 3,2)$, sendo muito próximo do valor esperado (conforme a otimização) que era de aproximadamente 3,3. Já o valor da relação r/l para a amostra OT foi de 0,19 , que, apesar de acima do valor restritivo $(0,15)$, não apresentou alta convexidade nem sobreposição lateral de cordão.

Pode-se dizer, então, que a repetibilidade experimental na obtenção dos cordões de amanteigamento foi comprovada e os valores otimizados de amplitude, tempo de parada lateral e velocidade de alimentação foram validados, obtendo-se cordões com alta relação ar/af (alta produção e baixa diluição) e convexidade satisfatória.

\section{Conclusões}

As predições pelas equações obtidas para ar/af e r/l, a partir do planejamento experimental adotado, possuem concordância com o esperado teoricamente. Naturalmente "ar" é governado apenas pela velocidade de alimentação (diretamente proporcional). Foi observada uma tendência do aumento da velocidade de alimentação diminuir "af", mostrando-se seu aumento ser favorável para a obtenção do cordão otimizado. Com o aumento na quantidade de material adicionado, o calor fornecido ao metal base é reduzido diminuindo a área fundida. Já o aumento do tempo de parada lateral tende a aumentar "af", uma vez que há uma permanência maior na lateral do cordão aumentando a largura, por isto seu valor deve ser mantido ao mínimo em amanteigamentos. Finalmente, o aumento da amplitude diminui a relação ar/af, mas por outro lado amplitudes baixas favorecem cordões com alta convexidade e com possibilidade de sobreposição lateral de cordão. Portanto, os valores de regulagem de parâmetros $\left(A=9,1 \mathrm{~mm} ; \mathrm{t}_{\mathrm{pl}}=0 \mathrm{~s}\right.$ e $\mathrm{V}_{\text {alim }}=52,5 \mathrm{~mm} / \mathrm{s}$ ) obtidos a partir da otimização da relação ar/ af, respeitando a restrição de $\mathrm{r} / \mathrm{l}<0,15$, é uma balanceamento favorável dos 3 fatores.

Conclui-se, pois, que a metodologia adotada de isolar os fatores de entrada de forma a só usar parâmetros independentes (utilizando a frequiência como parâmetro de ajuste para manter a velocidade transversal constante) foi capaz de identificar o efeito de cada parâmetro livre de tecimento e que os parâmetros obtidos podem ser adotados para outras deposições onde se deseja amanteigar de forma otimizada.

\section{Agradecimentos}

Ao CNPQ pelo fornecimento de bolsas de estudo para doutoramento e bolsa de pesquisa. Ao Centro para Pesquisa e Desenvolvimento de Processos de Soldagem (Laprosolda) da UFU pelo oferecimento de toda infra-estrutura laboratorial para realização dos experimentos. À SANDVIK pela doação do rolo de arame AWS ER 309L e à White Martins Gases Industriais pelo fornecimento do gás de proteção.

\section{Referências}

[1] FILHO, I. G. S. Estudo da influência dos parâmetros de soldagem de revestimento resistente à corrosão na diluição pelo processo TIG modalidade arame-quente. Dissertação de Mestrado, Universidade Tecnológica Federal do Paraná, Curitiba, 2007.

[2] LINNERT, G. E. Welding Metallurgy - Carbon e Alloy Steels. Miami: American Welding Society, ed. 4, p. 628, 1995.

[3] SCOTTI, A.; FERRARESI, V. A. Recuperação de uma engrenagem de ferro fundido em indústria de couro. Manutenção, no. 45, ABRAMAN, p. 36-39, 1993.

[4] SCOTTI, A. Process Modelling to Establish Control Algorithms for Automated GMAW. PhD Thesis, Cranfield Institute of Technology, School of Industrial and Manufacturing Science, UK, 1991.

[5] BARRA, S. R. Influência dos Procedimentos de Soldagem sobre a Resistência à Cavitação de Depósitos Obtidos com a Utilização de Arames Tubulares de Aços Inoxidáveis Ligados ao Cobalto. Dissertação de Mestrado, Universidade Federal de Santa Catarina, Florianópolis, 1998.

[6] LIMA, A. C. Estudo da Aplicação de Revestimento Duro por Soldagem por Arames Tubulares Quanto à Resistência ao Desgaste de Facas Picadoras de Cana-de-Açúcar. Tese de Doutorado, Universidade Federal de Uberlândia, Uberlândia/ MG, 2008. 